\title{
Telemedicine in Critical Care
}

\author{
Gastón Murias $^{1}$, Bernat Sales ${ }^{2}$, Oscar Garcia-Esquirol ${ }^{3}$ and Lluis Blanch ${ }^{* 4}$ \\ ${ }^{1}$ Intensive Care Units of Clínica Bazterrica and Clinica Santa Isabel, Buenos Aires, Argentina \\ ${ }^{2}$ Institut Universitari Fundació Parc Tauli, Corporació Parc Taulí, Universitat Autònoma de Barcelona, Parc Taulí s/n, \\ 08208, Sabadell, Spain \\ ${ }^{3}$ Critical Care Center, Hospital de Sabadell, Corporació Parc Taulí, Universitat Autònoma de Barcelona, Parc Taulí \\ s/n, 08208, Sabadell, Spain \\ ${ }^{4}$ Critical Care Center, CIBER Enfermedades Respiratorias (CIBERes), Hospital de Sabadell, Corporació Parc Taulí, \\ Universitat Autònoma de Barcelona, Parc Tauli s/n, 08208, Sabadell, Spain
}

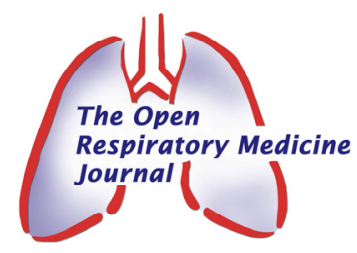

\begin{abstract}
Critical care medicine is the specialty that cares for patients with acute life-threatening illnesses where intensivists look after all aspects of patient care. Nevertheless, shortage of physicians and nurses, the relationship between high costs and economic restrictions, and the fact that critical care knowledge is only available at big hospitals puts the system on the edge. In this scenario, telemedicine might provide solutions to improve availability of critical care knowledge where the patient is located, improve relationship between attendants in different institutions and education material for future specialist training. Current information technologies and networking capabilities should be exploited to improve intensivist coverage, advanced alarm systems and to have large critical care databases of critical care signals.
\end{abstract}

\section{INTRODUCTION}

In many areas of medicine, physicians can often postpone decisions about patient care until they have been debated in committees; however, in critical care medicine, physicians must constantly take both general and specific decisions. Moreover, critical patients are nearly always undergoing different treatments and procedures. Although timely decisions are required for both decisions and procedures in critical care, the type of training that will enable the physician to reach them is different in each case. Short-term, specific training will enable staff to make procedures; however, the clinical skills and judgment required for decision making can only be acquired with years of experience.

Closed intensive care units (ICUs), i.e. those in which critical care specialists take charge of patients, have better outcomes than open units [1]. Thus, one might expect closed units to gradually replace open units. However, a survey of intensive care chiefs in Canada revealed that no specialists were present in about $50 \%$ of the units and that specialists were only present a few hours each day in another $25 \%$. In fact, $90 \%$ of the units did not meet the staffing standards recommended by the Society of Critical Care Medicine [2]. This situation is apparently worse in the United States [3, 4].

There are several reasons for this problem. First and foremost, there is a dire shortage of critical care specialists. To make matters worse, even if enough specialists were available to staff intensive care units, it is unlikely that the system could afford it. This makes it extremely difficult for

*Address correspondence to this author at the Critical Care Center, Hospital de Sabadell, Corporació Parc Taulí, 08208 Sabadell, Spain;

Tel: +34937458323; E-mail: lblanch@tauli.cat most hospitals to implement a model in which an intensivist is always present in the unit. And, given unparalleled growth in the demand for intensive care coupled with limited or null growth in the number of intensivists, this situation will only become worse and worse unless there is a profound change in the current paradigm [5].

However, the problem begins far before admission to the ICU. Critical care often begins in an ambulance. The emergency department is the main route of access to the hospital: excluding admissions for births, 55\% of all hospital admissions go through emergency departments [6].

In this scenario, one might expect more resources to be assigned to emergency departments to counterbalance increasing demand for emergency services. Unfortunately, this has not happened. In fact, budgetary limitations have even led to reduced funding [7-11].

Organizational problems also abound. Transfer teams are poorly integrated with hospital emergency departments, leading to inefficient use of the available resources and delays in diagnosis and treatment that can cost thousands of lives. For example, more than half a million ambulances are turned away from emergency departments in the United States each year [12].

Medical emergencies are a growing problem. Statistics from the Department of Health of the United Kingdom show that emergency consultations have increased at an annual rate of $6 \%$ since 1994 . To deal with this growth, diverse strategies have been employed with the aim of optimizing the use of resources. One such strategy involved changing from a first-in first-out system, where patients are visited in the order in which they arrive, to a system that assigns priority in function of the patient's degree of emergency. This 
solution, however, is not without problems. In a scenario with limited resources, faster attention to emergencies leads to delays in attending the remaining patients. Since the population became aware of this system of prioritization, emergency calls have increased $8 \%$ yearly (about $30 \%$ more than visits to the emergency department). This means that false emergency calls, practically unheard of in the mid 1990s, now account for about $20 \%$ of all emergency calls (1.1 million false emergencies in 5.6 million calls per year) [13].

Another, compounding problem is that emergency departments also suffer from a shortage of qualified staff, so that the number of units staffed by physicians has slowly decreased.

The impact of delays in attending trauma victims has been known for nearly a century. An analysis of the mortality of French soldiers in the first world war showed that $10 \%$ of the soldiers who were resuscitated within an hour of being wounded died compared to $75 \%$ of those who were resuscitated 8 hours after being wounded [14]. These data seem to have influenced Dr. R Adams Cowley in postulating the "golden hour" concept. Even if a particular time frame does not seem to be based on hard evidence [15]. It is clear that delays in resuscitation have a profound impact on outcomes. With time, evidence has accumulated that rapid attention is critical not only for trauma victims but also for cardiovascular emergencies, neurological emergencies, acute attacks in chronic respiratory disease, and sepsis.

In June 2006, after three years' work, a commission designated by the United States Institute of Medicine presented the results of a study about the future of medical emergencies [16-18]. Their principal recommendations could be summarized in four points:

- Integration of the different stages in patient care. Care for patients with medical emergencies, from the prehospital stage to hospital discharge, should be integrated, and changes from one stage to another should ideally be imperceptible.

- $\quad$ Proper channeling. Not all hospitals are appropriate for all patients: both the needs of the patient and the specialties and services available at a hospital vary widely. A system that works correctly should refer each patient to the most appropriate institution available.

- Guarantee of best practice according to current knowledge. In each stage of care, the patient should receive the best treatment available. This involves two principal aspects: not only is it necessary to guarantee what is done but also when it is done.

Possibility of evaluating the results of the system. The system can be considered to be working properly only if it can be shown that its results meet expected quality standards. Therefore, the system should be planned so that its results can be easily measured and compared. Ideally, the system should contain tools to enable the cause of errors and malfunctioning to be determined so that they can be corrected.

\section{THE PROBLEM OF RISING COSTS}

When healthcare expenditures are analyzed, two points become clear:

- $\quad$ These expenditures are astronomical and they represent a very high percentage of the GNP in all the systems studied.

- $\quad$ These expenditures are rising faster than ever.

Healthcare expenditures in the United States increased by $6.9 \%$ in the year 2007 (twice the rate of inflation), reaching 2.3 trillion dollars, which represents $16 \%$ of the GNP. Although these figures seem alarming, future projections are much worse. It is estimated that by 2016 healthcare expenditures will reach 4.2 trillion dollars; if the economy grows at the rate predicted when the growth in expenditures was estimated (early 2008), healthcare expenditures will represent $20 \%$ of the GNP (and a much higher percentage if the current financial-economic crisis results in a recession)[19]. This is a problem not only for the national budget but also for the individual worker's budget: in 2007, the average worker spent $\$ 1400$ more on healthcare than in 2000 [20]. Unless a radical change occurs, in 2008 the current trend will result in healthcare expenditures that surpass families' ability to save, placing families in debt or forcing them to reduce expenditures in other areas [21]. In a survey conducted by the Wall Street Journal and NBC, nearly half of those surveyed named the cost of healthcare as their main concern [22].

Studies show that the main reason that people give up medical coverage is the high cost involved [20] and economists have found a good correlation between increases in healthcare costs and decreases in medical coverage in the population [23].

To explain these disproportionate costs (especially in a system where 47 million people have no medical coverage [24], numerous arguments have been put forth: inefficiency, excessive administrative costs, poor administration, inadequate care (with an enormous increase in costs due to medical error), waste of resources, and fraud. In the analysis of avoidable expenditures, apart from the need to develop a more efficient system with lower administrative costs, one point that stands out is the need to reduce medical error. A report from the United States Institute of Medicine [25] shows the severity of the problem: not only does medical error kill between 44, 000 y 98, 000 persons per year in the United States (where 43, 458 deaths were attributable to vehicular accidents and 42, 297 to breast cancer), but it also costs the system between 17 and 29 billion dollars per year.

Although the system in the United States seems highly inefficient (the United States spends six times more money per capita on the administration of healthcare resources in comparison to countries in Western Europe [26] the situation is also alarming in other developed countries. Healthcare costs represent $10.9 \%$ of the GNP in Switzerland, $10.7 \%$ in Germany, 9.7\% in Canada, and 9.5\% in France [24].

A bed in the ICU costs eight times as much as bed in a general ward [27] and personnel costs account for $64 \%$ of 
these costs [28]. Considering these points, it is evident that with the current paradigm, the shortage of specialists is not the only reason why ICU staffing often fails to meet the standards proposed in international recommendations: even if enough specialists were available, the system probably would not be able to pay them.

The care and monitoring of critical patients in critical care units (ICU), operating rooms and emergency departments have evolved in the framework of the model in which the specialist is present in the same place as the patient. The specialist must be physically near the patient not only to perform medical acts and procedures but also to monitor the patient's evolution, deal with monitor alarms, access the clinical history, see the medical images, and finally take the appropriate decisions. Whereas a physician must be physically present to perform procedures such as placing a central venous line or intubating a patient, there is no reason why the rest of the physician's responsibilities cannot be fulfilled from a remote location. Moreover, considering that the level of skills and training necessary for these two types of tasks are clearly different, there is no reason why the same person should be in charge of carrying out both kinds of tasks. Thus, the shortage of specialists might give rise to an alternative arrangement. In this model, a paramedic or physician-intraining is physically present with the patient while a specialist is on call to assist with those problems that go beyond the training and experience of the professional who is present. This model has been implemented alone or as a complement to the traditional arrangement (usually to cover nights, weekends, and holidays). While this is a good alternative for dealing with a complex task in a less-than-ideal situation, it has at least two obvious flaws:

1. The staff present with the patient must be able to detect a problem to be able to call for help. Paradoxically, detecting problems is one of the tasks that require the most training.

2. When the specialist is called in, he or she must take decisions on the basis of clearly limited sources of information. Elements like the shape of the airway pressure curve, the variation in pulse pressure, the profile of the capnogram, the ventilatory pattern, the medical images, the EKG can only be considered through information transmitted by staff with less training. This increases the risk that potentially valuable information will be lost and adds to the level of uncertainty associated to the decision.

The second problem can be solved by changing the type of connection between the specialist and the less experienced staff physically present with the patient. If the voice connection is replaced or supplemented by a data connection, it is possible to transmit all the information necessary to enable decision making in a way that closely resembles the handson paradigm.

Telemedicine can also help resolve the first of the two problems. Intelligent aggregating (using automatic processes and intelligent alarms) could alert the specialist to potentially dangerous changes in the patient's status even when less experienced staff would not be able to detect a problem.

In addition to the direct advantages discussed above, telemedicine can bring indirect benefits to this scenario. The most refined telemedicine systems should integrate information from the hospital information system, including the data from monitoring devices. The acquisition and standardization of these signals would dramatically increase the semantic value of medical registers. More importantly, this information could be used for other purposes.

\section{CURRENT ALARM SYSTEMS}

Whereas only $10 \%$ of patients admitted to ICUs require major interventions, $77 \%$ are admitted solely for monitoring purposes [29], and ICUs are equipped with devices that can monitor physiological variables of interest. As the response time to dangerous events is crucial, monitoring systems normally sound an alarm to alert staff to potentially risky situations. Despite technological advances in this area, the situation remains far from ideal:

The technologies used by monitoring systems produce signals with variable signal-to-noise ratios (SNR), which means that the reproducibility of these signals is also variable. Furthermore, device manufacturers' main concern is to eliminate false negatives (in which a true event goes undetected) while false positives (sounding an alarm when there is no real risk to the patient) are accepted as a necessary though annoying evil. However, the combination of a low threshold for alarms and noisy signals has generated a serious problem in critical care units. Even in the operating room, where patients are usually deeply sedated and often under the effects of muscle relaxants, Kestin et al. found that only $3 \%$ of the alarms corresponded to an event that represented a real risk to the patient [30]. The situation is worse in the ICU, where patients tend to be awake and occasionally agitated (producing noisier signals). In a pediatric ICU, Lawless [31], found that less than $6 \%$ of the 2, 176 alarms activated during the observation period were clinically important, yielding a positive predictive value of $7 \%$ for pulse oximeters, $16 \%$ for capnographs, $3 \%$ for ventilators, and $5 \%$ for EKGs. With an average of 4.3 devices with monitoring capabilities per patient [32], and up to 10 alarms per device [33], the magnitude of the problem is evident. Three aspects are of particular concern:

1. The frequency of false alarms is probably one of the reasons why the response to alarms is often delayed (or nonexistent). In 2002, the Joint Commission on Accreditation of Health Care Organizations reviewed 23 cases of death or severe lesions associated to mechanical ventilation: they found that the problem was related to delayed attention to alarms or failure of alarms to activate due to inappropriate parameter settings in $65 \%$ (not a single case of equipment malfunctioning was detected) [34]. One survey of 23 anesthesiologists and vascular surgeons [35] found that only heart rate and blood pressure alarms were configured (apparently the rest were turned off) and with such extreme limits that even definitely dangerous situations would not be detected. Moreover, even in this scenario, personnel tolerated alarms sounding for as long as 10 minutes before responding [35].

2. ICU patients and staff are exposed day and night to noise levels above the $45 \mathrm{Db}$ recommended by the Environmental Protection Agency, with peaks of $90 \mathrm{Db}$ (typical of measurements taken $10 \mathrm{~m}$ from a ma- 
jor highway) [36]. This has been related to the poor quality of sleep of ICU patients [37], and burnout in ICU nurses [38].

3. Staff attending false alarms costs money.

\section{SOPHISTICATED ALARM SYSTEMS}

Incorporating more complex algorithms into current monitoring systems could substantially alleviate the problems caused by alarms:

1. To date, signals have been treated as if they were unrelated. Even though the signals from the EKG and respiratory plethysmography are measured by the same sensors, malfunction of one of the electrodes usually sets off a low-level EKG alarm (electrode disconnected) and a maximum level plethysmographic alarm ("Apnea").

2. Furthermore, a specialist rarely considers data from a single device in isolation. Understanding physiological and pathophysiological phenomena requires a more global vision: considering bradycardia, hypertension, and increased intracranial pressure together provides much more information than sum of its parts. Although each of these elements can result from multiple causes, the combination of the three alterations drastically reduces the level of uncertainty and points to the Cushing reflex. Once again, having the signals available for measurements, calculations, and inferences brings added value in telemedicine.

3. Occasionally, temporal variations in vital parameters provide more information than the parameter alone. A heart rate of $90 \mathrm{bpm}$ is completely normal in some circumstances; however, if it was $60 \mathrm{bpm}$ an hour earlier, there may be cause for concern.

An advanced telemedicine system that can acquire signals from different devices would make it possible to increase the degree of integration.

Once the information is acquired from the monitoring devices, alarms are no longer limited to sounds emitting from a patient's bedside (or from the nursing station). The telemedicine device can employ diverse gateways to send alarms to e-mail addresses, paging devices, cell phones, etc. Moreover, in addition to alerting a remote specialist to a potentially dangerous event, a system of scalable alarms can come into effect if the problem is not solved within a determinate time frame--for example, sending an SMS to a supervisor (or activating the remote monitoring system).

\section{Specialists' Recommendations}

The final aim of programming an alarm is to improve patients' safety. However, in an environment where qualified human resources are scare, merely sounding an alarm about a potentially dangerous event does not guarantee that this objective will be met. Thus, incorporating medical information acquired from specialists into monitoring systems could help us understand these phenomena and suggest solutions for these problems. The level of integration achieved and the quality of the structuring of the information in the medical records will determine the usefulness of the information acquired.

\section{Calculating Parameters}

Sometimes, it can be very useful to combine elements from different monitoring devices. Measuring the volume of expired $\mathrm{CO} 2$ (rather than the pressure of $\mathrm{CO} 2$ in expired gases, usually available) enables the approximate state of the patient's metabolism to be estimated, and some parameters calculated from volumetric capnography provide information about the condition of the lungs and cardiovascular function. If the information from the ventilator flow sensor were combined with information from the patient's capnograph (nearly always available in mechanically ventilated patients), information about the volume of expired $\mathrm{CO} 2$ would be easily available without the need for additional equipment.

\section{Automatic Curve Analysis}

Current monitoring systems show waves and values. Although alarms typically sound in function of values, analyzing curves can provide much more information. For instance, the appearance of a $\mathrm{P} 2$ wave greater than the $\mathrm{P} 1$ wave in the intracranial pressure trace can alert to a decrease in intracranial compliance, persistent airway flow at end-expiration can confirm the presence of autoPEEP, a marked variation in the pulse pressure can suggest the usefulness of intravenous fluid loading, etc. Specialists usually analyze curves at the bedside, but making sense of curves normally requires extensive training. However, once the information from the signals from the devices has been acquired by a telemedicine system, it is relatively simple to implement automatic processes to evaluate these phenomena and the information. For this purpose, it is important for the information to be available in a format independent of the manufacturer of the monitor or ventilator.

\section{INTEGRATING TELEMEDICINE AND E-LEARNING SYSTEMS}

At least nowadays, one part of staff training - the part centered on the acquisition of skills - requires the physical presence of both teacher and pupil. However, much training involves the acquisition of the theoretical knowledge that makes up most of the curriculum, and this objective can be accomplished equally through either traditional or eLearning arrangements.

Given the potential consequences of medical errors, healthcare professionals receive extensive training and are expected to maintain errors to a minimum. Case-based learning systems (medical residency programs, for example) yield the best results. However, these systems are labor intensive and consume enormous amounts of resources and time. Furthermore, this kind of learning can increase patient morbidity and mortality. This is where e-Learning can help most. Computer simulations can replicate the intellectual processes involved in case-based learning.

In the late 1990s, Brandon Hall, editor of e-Learning Magazine, predicted that $50 \%$ of training in 2003 would involve some kind of electronic training. According to the International Data Corporation, in the United States alone, the volume of e-Learning business in 2003 was over 11 billion dollars, reflecting a sustained annual growth of approximately $83 \%$ over a five-year period. Although the corporative sector accounts for most of this growth, the advent of cheaper, more powerful computers, together with more and 
better specific programs, has brought these tools to the academic environment.

E-Learning comprises many different strategies with very different levels of technological development. At one end of the spectrum, e-Learning is nothing more than the delivery of electronic documents for office applications (text files, electronic forms, slide presentations). In this approach, computers merely change the way contents reach students; the characteristics of the learning process remain unchanged. At the other end of the spectrum, sophisticated simulation systems allow the rules of the process to be changed, facilitating case-based learning. Unfortunately, in most simulations systems the number of cases that can be introduced is limited during the programming stage.

Integrating telemedicine and e-Learning systems could be highly advantageous. The system would make it possible to save interesting cases and to construct a teaching library. Cases in the library might include information about the problem and solutions tested to enable the posterior analysis of the actions undertaken and the effects brought about by different maneuvers or changes. Thus, rather than simulations, this approach would enable learning based on real cases as well as on the mistakes made.

Cases could be enriched by attaching notes, commentaries, summaries from the clinical history, audio layers with messages including recommendations from specialists, etc. to the registers (to the extent that these elements can be aggregated as superimposed layers, the original registers remain unchanged).

All the information about the use of the material (viewing, commentaries, invitations, questions) could be registered and ready to be exported to any e-Learning system.

\section{TECHNICAL REQUIREMENTS}

\section{Communication Channel}

A communication channel is a medium through which information is transmitted between two points. Some characteristics of the communication channel determine the functionalities that can be expected from a telemedicine system.

Bandwidth of the channel is its capacity or the amount of data that the medium can theoretically transmit in a given time. As available bandwidth is not unlimited, any application that transmits information between two points must be designed with these limitations in mind. The ideal system is scalable and adaptable to the capacity of the different channels through which the system functions; in other words, it is a system that is capable of taking advantage of the maximum capacity of the medium used for the transmission of the information.

Dataflow is the continuity or fluidity of transmission during the communication. The flow measures the real performance of the channel; it depends on the bandwidth and the delay in delivering the information that the medium can guarantee in practice. Dataflow is even more important than bandwidth: in systems that transmit information in real time, in which constant updating is crucial, flow is what really determines the suitability of the channel. Whereas dataflow in fixed networks generally has upper and lower limits, the problem is worse in mobile networks because it is practically impossible to guarantee a minimum dataflow due to aspects like coverage or network congestion. Therefore, systems that function through mobile networks must be designed with the limitations of this medium in mind, such as oscillations in the dataflow of the channel and even loss of the connections.

Telemedicine systems handle sensitive data that must operate on channels that can guarantee the security and privacy of the information. There are diverse methods to maintain the security of a system, among others, the use of virtual private networks (VPN), encryption techniques, or certificates and digital signatures.

\section{Accuracy}

In any system for the practice of telemedicine, the value of the information transmitted depends on its quality, representation, and reliability. In these critical systems, all data with questionable quality, representation, or reliability are worse than worthless because they could lead to medical errors and consequent risks. Both the channel and the system used are essential for ensuring the validity of the information in these terms.

Whenever waves and not only isolated values are transmitted, it becomes essential to identify the minimal information that defines the profile and characteristics of the curves. The characteristics of current communication channels (with the corresponding restrictions regarding capacity and dataflow) make it essential to determine the minimum volume of data required for a complete and unequivocal representation of the waves transmitted.

\section{Interface}

It is equally important to present the information in a way that facilitates the reading of these curves. The telemedicine system's interface, the nomenclature of the waves, and the type of presentation should be homogeneous; therefore, they should be independent of the devices generating the signals. In these cases, it is best if the system handles the homogenization of these signals and their processing (their interpolation and the adjustment of the different clocks) to ensure a clear, totally synchronized presentation that reliably reproduces the original curves. Another desirable factor to improve readability and facilitate comprehension is the integration of diverse sources under the same interface. This can be achieved by constructing systems capable of handling all the tasks or, more frequently, of guaranteeing the communications among different systems responsible for solving punctual problems.

\section{Standards}

Communications among different systems can be ensured through standards for the storage and communication of data. Although its implementation is far from complete, HL7 (Health Level 7) is a set of standards for the electronic exchange of medical information that will probably become the standard in telemedicine. HL7 encompasses clinical and administrative information; its most commonly used specification is a messaging standard for the electronic interchange of healthcare data between systems and subsystems on the one hand, and between these and the hospital information system (HIS) on the other. This makes it possible, for example, to assign specific tests or results to a specific patient in the HIS 
or in the corresponding system. The storage standard is even less well defined, with the exception of the DICOM (Digital Imaging and Communication in Medicine) format, which is widely used for images. Although DICOM was initially created for the management, storage, printing, and transmission of medical images, its definitions have been expanded to include other types of data, such as physiological waves (DICOM Waveform). Taking into account DICOM's enormous level of penetration and the number of viewers available, it is likely to become the storage standard for other data apart from medical images.

Synchronizing capture and ensuring sufficient definition not only help make all these signals easier to understand, they are also essential to enable these signals to be used for research, teaching, expert systems, and data mining.

\section{CONCLUSIONS AND IMPLICATIONS}

Telemedicine has obvious advantages: it enables specialists to attend patients that need them in situations where human resources are unavailable or economically unviable. Nevertheless, these direct advantages can even be surpassed by the indirect advantages related to the possibility of acquiring, normalizing, synchronizing, and storing medical signals. In the very near future, it will be possible to amplify the reach of alarm systems, improve their specificity, and enable the setting off of qualitatively different alarms: in addition to emitting sounds at the patient's bedside, e-mails, SMS, or pager messages can be sent immediately or according to a predefined scaling system when problems are not satisfactorily solved. Not only will this change the way medical knowledge is applied, but also they way knowledge itself is produced. Applying data-mining techniques to large databases of medical signals can generate huge amounts of information and lead to a new strategy for formulating hypotheses to be tested with the scientific method. Furthermore, this would make it possible to explore the signals in ways that are impossible with the naked eye, such as signal analysis in different temporal domains, of which frequencies are probably the most widely known. It is even reasonable to expect that these analyses might reveal systematic alterations in one or more signals before the onset of an event. Thus, we can consider the possibility of emitting alerts about events that might occur as well as those that have already occurred.

\section{REFERENCES}

[1] Carson SS, Stocking C, Podsadecki T, et al. Effects of organizational change in the medical intensive care unit of a teaching hospital: a comparison of 'open' and 'closed' formats. JAMA 1996; 276(4): 322-8.

[2] Parshuram CS, Kirpalani H, Mehta S, Granton J, Cook D. In-house, overnight physician staffing: a cross-sectional survey of Canadian adult and pediatric intensive care units. Crit Care Med 2006; 34(6): 1674-8.

[3] Groeger JS, Strosberg MA, Halpern NA, et al. Descriptive analysis of critical care units in the United States. Crit Care Med 1992; 20(6): 846-63.

[4] Angus DC, Shorr AF, White A, Dremsizov TT, Schmitz RJ, Kelley MA. Critical care delivery in the United States: distribution of services and compliance with Leapfrog recommendations. Crit Care Med 2006; 34(4): 1016-24.

[5] Angus DC, Kelley MA, Schmitz RJ, White A, Popovich J, Jr. Caring for the critically ill patient. Current and projected workforce requirements for care of the critically ill and patients with pulmonary disease: can we meet the requirements of an aging population? JAMA 2000; 284(21): 2762-70.
Owens PEA. Hospital admissions that began in the emergency department, 2003. Agency For Healthcare Research And Quality, Statistical Brief; Rockville: Md, 2006; vol. 1.

[7] Schafermeyer RW, Asplin BR. Hospital and emergency department crowding in the United States. Emerg Med (Fremantle) 2003; 15(1): 22-7.

[8] American Hospital Association. Emergency departments: an essential access point to care. Trendwatch 2001; $3: 1-8$.

[9] Lewin Group. Emergency department overload: a growing crisis: the results of the aha survey of emergency department (ed) and hospital capacity. American Hospital Association, Falls Church, VA 2002.

[10] Brewster LR, Rudell LS, Lesser CS. Emergency room diversions: a symptom of hospitals under stress. Issue Brief Cent Stud Health Syst Change 2001; 38: 1-4.

[11] US General Accounting Office. Hospital emergency departments: crowding conditions vary among hospitals and communities. Washington, DC: US General Accounting Office, 2003. Available at: http://www.gao.gov/new.items/d03460.pdf

[12] Burt CW, McCaig LF, Valverde RH. Analysis of ambulance transports and diversions among US emergency departments. Ann Emerg Med 2006; 47(4): 317-26.

[13] Health and Social Care Information Centre (UK). Ambulance services, England: 2004-05. Departament of Health; UK 2005.

[14] Santy P, Marquis Moulinier D. Shock tramatique dans les blessures de guerre, analysis d'observations. Bull Med Soc Chir 1918; 44: 205.

[15] Lerner EB, Moscati RM. The golden hour: scientific fact or medical "urban legend"? Acad Emerg Med 2001; 8(7): 758-60.

[16] Committee on the future of emergency care in the U.S. hospital based emergency care at the breaking point. Washington, DC: National Academy Press; 2007. Available at: http: //www.nap.edu/ catalog.php?record_id $=11621$

[17] Committee on the future of emergency care in the U.S. emergency medical services at the crossroads. Washington, DC: National Academy Press; 2007. Available at: http: //www.nap.edu/ catalog.php?record_id $=11629$

[18] Committee on the future of emergency care in the U.S. emergency care for children: growing pains. Washington, DC: National Academy Press; 2007. Available at: http: //www.nap.edu/catalog.php?record_id $=11655$

[19] Poisal JA, Truffer C, Smith S, et al. Health spending projections through 2016: modest changes obscure part D's impact. Health Aff 2007; 26(2): W242-53.

[20] Gary CG, DiJulio B, Finder B, et al. Employer health benefits 2007 annual survey. Chicago, Il: The Kaiser Family Foundation and Health Research and Educational Trust, 2007. Available at: http: //www.kff.org/insurance/7672/upload/76723.pdf

[21] McKinsey and Company. Will health benefit costs eclipse profits? [Web Page]. September 2004; Available at: http: //www.mckinseyquarterly.com/newsletters/chartfocus/2004_09.htm

[22] Harwood J. America's economic mood: gloomy. Wall Street J 2007 (Health).

[23] Kaiser Commission on Medicaid and the Uninsured. The Uninsured: A primer. key facts about americans without health insurance. Washington, DC: The Henry J. Kaiser Family Foundation, 2006. Available at: www.kff.org/uninsured/upload/7451.pdf

[24] California Health Care Foundation. Snapshot: Health Care Costs 101. Oakland, CA: California Health Care Foundation, 2005. Available at: http: //www.chcf.org/documents/insurance/HCCosts 10105.pdf

[25] Institute of Medicine of the National Academies. To err is human: building a safer health system. Washington, DC: National Academies Press, 1999. Available at: http: //www.nap.edu/openbook. php?isbn $=0309068371$

[26] Angrisano C, Farrell D, Kocher B, Laboissiere M, Parker S. Accounting for the cost in the United States. Sao Paulo, Brazil: McKinsey and Co. 2007. Available at: http: //www.mckinsey.com/ $\mathrm{mgi} / \mathrm{rp} /$ healthcare/accounting_cost_healthcare.asp

[27] Wagner DP, Wineland TD, Knaus WA. The hidden costs of treating severely ill patients: charges and resource consumption in an intensive care unit. Health Care Financ Rev 1983; 5(1): 81-6.

[28] Noseworthy TW, Konopad E, Shustack A, Johnston R, Grace M. Cost accounting of adult intensive care: methods and human and capital inputs. Crit Care Med 1996; 24(7): 1168-72. 
[29] Thibault GE, Mulley AG, Barnett GO, et al. Medical intensive care: indications, interventions, and outcomes. N Engl J Med 1980; 302(17): 938-42.

[30] Kestin IG, Miller BR, Lockhart CH. Auditory alarms during anesthesia monitoring. Anesthesiology 1988; 69(1): 106-9.

[31] Lawless ST. Crying wolf: false alarms in a pediatric intensive care unit. Crit Care Med 1994; 22(6): 981-5.

[32] Deller A, Schuhle B, Konrad F, Kilian J. Alarms of medicaltechnical equipment in the surgical intensive care unit: a prospective study. Anasth Intensivther Notfallmed 1988; 23(5): 238-43.

[33] Kacmarek RM, Meklaus GJ. The new generation of mechanical ventilators. Crit Care Clin 1990; 6(3): 551-78

[34] Joint Commission on Accreditation of Health Care Organizations. Sentinel event alert. Preventing ventilator-related deaths and injuries.
Washington, DC: Joint Commission 2002. Available at: http: //www. jointcommission.org/SentinelEvents/SentinelEvent Alert/sea 25.htm

[35] Koski EM, Makivirta A, Sukuvaara T, Kari A. Clinicians' opinions on alarm limits and urgency of therapeutic responses. Int J Clin Monit Comput 1995; 12(2): 85-8.

[36] Balogh D, Kittinger E, Benzer A, Hackl JM. Noise in the ICU. Intensive Care Med 1993; 19(6): 343-6.

[37] Aaron JN, Carlisle CC, Carskadon MA, Meyer TJ, Hill NS, Millman RP. Environmental noise as a cause of sleep disruption in an intermediate respiratory care unit. Sleep 1996; 19(9): 707-10.

[38] Topf M, Dillon E. Noise-induced stress as a predictor of burnout in critical care nurses. Heart Lung 1988; 17(5): 567-74.

Received: December 22, 2008

Revised: January 2, 2009

Accepted: January 20, 2009

(C) Murias et al.; Licensee Bentham Open.

This is an open access article licensed under the terms of the Creative Commons Attribution Non-Commercial License (http://creativecommons.org/licenses/by$\mathrm{nc} / 3.0 /$ ) which permits unrestricted, non-commercial use, distribution and reproduction in any medium, provided the work is properly cited. 